КОСМИЧЕСКАЯ ФИЗИКА

КИНЕМАТИКА

И ФИЗИКА

НЕБЕСНЫХ

ТЕЛ том 28 № 52012

UDC 52-337

\author{
B. A. Shakhov ${ }^{1}$, M. Jurcisin ${ }^{2}$, E. Jurcisinova ${ }^{2}$, M. Stehlik ${ }^{2}$ \\ ${ }^{1}$ Main Astronomical Observatory of NASU, Zabolotnoho Str. 27 Kyiv, Ukraine, 03680 \\ ${ }^{2}$ Institute of Experimental Physics of SAS, 04001 Kosice, Watsonova 47, Slovakia \\ stehlik@saske.sk
}

\title{
The spontaneous magnetic field direction in an anisotropic MHD dynamo
}

The phenomenon of magnetic field generation in an astrophysical plasma in the frame of developed magnetohydrodynamic (MHD) turbulence is considered. The functional quantum field renormalization group approach is applied to helical anisotropic MHD developed turbulence which is stabilized by the self-generated homogeneous magnetic field. The purpose of the study is to calculate the value as well as direction of the magnetic field in the stochastic dynamo model. The generated magnetic field is determined by ignoring divergent rotor part of Green function of the magnetic field. It is shown that the magnetic field direction is connected with unique existing vector $\boldsymbol{n}$ describing the anisotropic turbulence forcing.

НАПРЯМОК СПОНТАННОГО МАГНІТНОГО ПОЛЯ У АНІЗОТРОПНОМУ МГД-ДИНАМО, Шахов Б. О., Юрчишин М., Юрчишинова Е., Стеглік М. - Розглядається явище генераџї̈ магнітних полів в астрофізичній плазмі у межах моделі розвиненої МГД-турбулентності. Застосовується квантовопольовий підхід ренормалізаційної групи до розвиненої гіротропно-анізотропної турбулентності, яка стабілізується самогенерованим магнітним полем. Метою ичього дослідження є обчислення як значення, так і напрямку магнітного поля у стохастичній моделі динамо. Генероване магнітне поле визначається при знехтуванні дивергентної роторної частини функиії Гріна магнітного поля. Показано, шуо напрямок магнітного поля пов'язаний з наявністю вектора $\boldsymbol{n}$, який описує анізотропне накачування енергії у турбулентність.

НАПРАВЛЕНИЕ СПОНТАННОГО МАГНИТНОГО ПОЛЯ В АНИЗОТРОПНОМ МГД-ДИНАМО, Шахов Б. А., Юрчишин М., Юрчишинова Е., Стеглик М. - Рассматривается явление генерации магнитных 
полей в астрофизической плазме в рамках модели развитой МГД-турбулентности. Применяется квантовополевой подход ренормализационной группь к развитой гиротропно-анизотропной турбулентности, которая стабилизируется самогенерируемым однородным магнитным полем. Целью этого исследования является вычисление как значения, так и направления этого магнитного поля в стохастической модели динамо. Генерируемое магнитное поле определяется при пренебрежении дивергентной роторной частью функиии Грина магнитного поля. Показано, что направление магнитного поля связано с наличием вектора $\boldsymbol{n}$, описывающего анизотропное накачивание энергии в турбулентность.

\section{INTRODUCTION}

Magnetohydrodynamic (MHD) turbulent dynamo still attracts large attention due to many applications in both the astrophysical and laboratory plasmas. Despite an enormous effort, nowadays the related problems and questions remain deficiently explored. That is question, how does the turbulence amplify and sustain magnetic fields (MFs), what is the spectrum and structure of this field at various scales in astrophysics as well as in a laboratory. Many authors attempt to justify the basic treatment of stable regimes of fully developed MHD turbulence in various approaches: kinetic or magnetic driving the helical or non-helical dynamo. Another eventual approach is based on the self-consistent nonlinear set of MHD equations, the Navier-Stokes equation including the Lorentz force together with the induction equation.

Nowadays the mean field helical dynamo is used to be applied in astrophysical rotators [22, 26, 28]. It involves initially weak large-scale field amplified by strong helical velocity fluctuations. In this understanding the large-scale field is amplified and sustained on scales significantly larger than the scale of the driving turbulence, and the source of $\mathbf{E}=\alpha \mathbf{B}(\alpha$ is known as the dynamo coefficient) typically is the kinetic helicity, $\mathbf{H}_{k} \equiv$ $\langle\mathbf{v} \cdot \operatorname{rot} \mathbf{v}\rangle$. Calculations of the field spectra are obviously performed in the case of isotropic MHD turbulence. Recently, anisotropic contributions from mean velocity flows have been considered as additional contributions to the electromotive force driving the velocity driven helical dynamo [4, 31]. Generally, the MHD turbulence is nowadays understood to be inherently anisotropic. A number of important physical processes in anisotropic MHD turbulence was clarified in [2, 17, 23, 24] where the concept of critical balance was used to determine the ratio of the dimensions of turbulent eddies in the directions parallel and perpendicular to the local MF.

As was emphasized in [6], the understanding of laboratory and/or astrophysical dynamo depends on dominant contribution (role) of magnetic fields, or alternatively, typical flows (known as the kinematic dynamo). In classical understanding of nonlinear astrophysical dynamo theory one separates "small-scale nonhelical dynamos" in which magnetic energy is ampli- 
fied by random force-line stretching on scales approximately less to that of the turbulent forcing $[16,20,21]$. Contrary to "large-scale helical dynamos", the helical hydrodynamic turbulence produces MF on scales larger than that of the input turbulent forcing (this is one we will consider here) [1, $5,7,22,26,28,29]$. This type of dynamo is often used to explain MFs of the solar and stellar coronae as well as coronae above accretion discs [14,15]. Numerical studies of the effect of a regular MF on $\alpha$-dynamo action, due to helicity driven MHD turbulence, are reported in [27] for unite Prandtl number. They found that the apparent effect of the dc-magnetic field is to suppress the dynamo action. Recently several papers deal with a special flow pattern, so-called "Roberts flow", which concerns the low-dimensional dynamo modeling $[12,32,37]$. This model can be useful for the examination of laboratory experiments. They attempt to solve numerically the dynamo problem in the Fourie representation with small discrete modes. Note that the kinetic helicity is not necessary for a modified version of the Robertstype dynamo [30].

In the helical MHD an additional problem arises: the instabilities induce the exponential increase of the magnetic fluctuations in the large scales range (see in [11], for example). The elimination of this instability leads to formation of a large-scale magnetic field known as the turbulent dynamo. Removal of the instability in quantum field formulation of helical MHD can be achieved by means of a nice and very well-known spontaneous symmetry breaking mechanism followed by the creation of a homogeneous stationary magnetic field. The problem was analyzed in [3] in the special case of isotropic MHD turbulence. Indeed, the basic physical problem arising in the MHD turbulence stability regime is that arising "rotor instabilities" in MHD turbulence needs the creation of a large-scale magnetic field, but then the MHD turbulence does not to be isotropic. For example, the Alfvénic turbulence (weak forcing when the turbulence excitations are small-amplitude disturbances propagating along the MF) is manifestly anisotropic [33].

The stabilization of an instable system through the appearance of a spontaneous mean field is a typical effect in field-theoretic models of various phenomena. A typical example is the appearance of spontaneous magnetization below the critical temperature $T_{c}$ in ferromagnetics. There, the standard technical procedure can be used. However, in the present problem this technique is not applicable (as was discussed in [3]). Here, we introduce a nonzero mean magnetic field already in origin problem to stabilize the turbulent system.

In $[10,13]$ the dynamo coefficient $\alpha$ was derived using the quasilinear MHD turbulent theory. They found that in the limit of large kinetic Re and magnetic $\mathrm{Re}_{m}$, the $\alpha$-coefficient may be not small if the correlation time of velocity field and magnetic field are shorter than the eddy turn over time of the MHD turbulence and $\alpha$ depends intensively on the magnetic Prandtl number. The opposite limit of small magnetic and fluid Reynolds numbers was considered in [35]. In this case there is no small-scale dynamo action and so the small-scale magnetic field is solely due to shredding the large- 
scale magnetic field. In general $\alpha$ has a tensor form, $\alpha_{i j}$ [22], which was also used in numerical simulations $[8,9]$. They showed rather fluctuating value of the $\alpha$-effect tensor in all the components. Thus the general case of an anisotropy presence is to be examined.

Here we will use the functional quantum field renormalization group $(\mathrm{RG})$ approach $[1,36]$. It assigns a field action to the stochastic problem and makes possible to use elegant and very well developed RG procedure in quantum field theory to investigate infrared asymptotic regimes of a stochastic system. This theoretic-field RG approach concerns to (describes) stationary state of generated MF under equilibrium in the sense when the state is the solution of self-consistent nonlinear MHD equations (in a helical medium) with the helical injection of energy. (In the RG terminology the stable regime is described by the fixed point of RG-equations.) Continuous injection of helicity typically leads to a quasi-steady dynamical equilibrium with self-generated MF as a stabilizing factor $[18,34]$. But in the isotropic developed MHD turbulence it is not possible to determine a direction of generated homogeneous MF. This question is the goal of the present paper. We use the theoretic-field RG approach to helical anisotropic MHD turbulence which is stabilized by the self-generated MF. Its direction is connected with unique existing vector $\mathbf{n}$ describing the anisotropic forcing (see below) .

\section{THE MODEL FORMULATION}

The interaction of electrically neutral conductive turbulent incompressible fluid (with the unit magnetic permeability) with the magnetic field in the case of anisotropically driven MHD turbulence is described by the MHD equations for the fluctuating part of the fields:

$$
\begin{aligned}
& \hat{L}_{v} \equiv-\partial_{t} \mathbf{v}+v \Delta \mathbf{v}+(\mathbf{v} \nabla) \mathbf{v}+(\mathbf{b} \nabla) \mathbf{b}+\mathbf{f}_{A}^{v}+\widetilde{\mathbf{f}}^{v}=\mathbf{f}, \\
& \hat{L}_{b} \equiv-\partial_{t} \mathbf{b}+v^{\prime} \Delta \mathbf{b}+(\mathbf{v} \nabla) \mathbf{b}-(\mathbf{b} \nabla) \mathbf{v}+\mathbf{f}_{A}^{b}+\widetilde{\mathbf{f}}^{b}=0 .
\end{aligned}
$$

The first equation is the well-known Navier - Stokes equation for the transversal velocity field $\mathbf{v}(\mathbf{x}, t)$ with the additional nonlinear contribution of the Lorentz force (the longitudinal contribution is ascribed to pressure $p$ ). The second equation for magnetic field $\mathbf{b}(\mathbf{x}, t)$ (in the Alfven units $\mathbf{b}=$ $\mathbf{B} / \sqrt{4 \pi \rho}, \rho$ is a fluid density) follows from the Maxwell equations for continuous medium. The magnetic diffusion coefficient $v^{\prime}$ is connected with the coefficient of molecular viscosity by relation $v^{\prime}=u v$ with dimensionless magnetic Prandtl number $u^{-1}$. The terms $\mathbf{f}_{A}^{i}$ are related to uniaxial anisotropy and they have the following form [2]:

$$
\begin{gathered}
\mathbf{f}_{A}^{v}=v\left[\chi_{1}(\mathbf{n} \nabla)^{2} \mathbf{v}+\chi_{2} \mathbf{n} \nabla^{2}(\mathbf{n v})+\chi_{3} \mathbf{n}(\mathbf{n} \nabla)^{2}(\mathbf{n v})\right]+ \\
+\lambda_{1} \mathbf{b}(\mathbf{n} \nabla)(\mathbf{n b})+\lambda_{2} \mathbf{n}(\mathbf{n} \nabla) b^{2}+\lambda_{3} \mathbf{n}(\mathbf{b} \nabla)(\mathbf{n b})+\lambda_{4} \mathbf{n}(\mathbf{n} \nabla)(\mathbf{n b})^{2}, \\
\mathbf{f}_{A}^{b}=v^{\prime}\left[\tau_{1}(\mathbf{n} \nabla)^{2} \mathbf{b}+\tau_{2} \mathbf{n} \nabla^{2}(\mathbf{n v})+\tau_{3} \mathbf{n}(\mathbf{n} \nabla)^{2}(\mathbf{n v})\right] .
\end{gathered}
$$


The parameters $\chi_{i}$ and $\tau_{i}$ characterize the weight of the individual structures in Eqs (2), and the unit vector $\mathbf{n}$ specifies the direction of the anisotropy axis.

The large-scale random force per unit mass $\mathbf{f}$ corresponds to a kinetic energy doping and it is assumed to have Gaussian statistics defined by the averages:

$$
\left\langle f_{i}\right\rangle=0,\left\langle f_{i}\left(\mathbf{x}_{1}, t_{1}\right) f_{j}\left(\mathbf{x}_{2}, t_{2}\right)\right\rangle=D_{i j}\left(\mathbf{x}_{1}-\mathbf{x}_{2}, t_{1}-t_{2}\right) .
$$

The two-point correlation tensor

$$
D_{i j}(\mathbf{x}, t)=\delta(t) \int d \mathbf{k}(2 \pi)^{3} \widetilde{D}_{i j}(\mathbf{k}) \exp (i \mathbf{k} \cdot \mathbf{x})
$$

is convenient to parametrize in the following way:

$$
\widetilde{D}_{i j}(\mathbf{k})=20 \pi^{2} g u v^{3} k^{1-2 \varepsilon}\left[\left(1+\alpha_{1} \xi_{k}^{2}\right) P_{i j}(\mathbf{k})+\alpha_{2} R_{i j}(\mathbf{k})+\rho Q_{i j}\right]
$$

as linear combination of both tensor and pseudotensor (in our helical case). Here $\varepsilon \geq 0$ is dimensionless parameter of the model; its physical value is $\varepsilon=2$ which corresponds to the Kolmogorov energy pumping from infra-red region of the small $\mathbf{k}$. The value $\varepsilon=0$ corresponds to a logarithmic perturbation theory for a calculation of Green functions when $g$, which plays the role of a bare coupling constant of the model, becomes dimensionless [36]. The problem of the continuation from $\varepsilon=0$ to the physical values was discussed in [1]. The $(3 \times 3)$-matrices $P_{i j}, R_{i j}$, and $Q_{i j}$ are the transverse projectors. Their explicit forms are defined by the relations (in the wave-number space):

$$
\begin{gathered}
P_{i j}(\mathbf{k})=\delta_{i j}-\frac{k_{i} k_{j}}{k^{2}}, \quad R_{i j}(\mathbf{k})=P_{i r}(\mathbf{k}) n_{r} n_{s} P_{s j}(\mathbf{k}), \\
Q_{i j}(\mathbf{k})=\mathrm{i} \varepsilon_{i j m} \frac{k_{m}}{k},
\end{gathered}
$$

where $\varepsilon_{i j m}$ is the Levi - Civita pseudotensor and $\xi$ is given by the equation $\xi=\mathbf{k} \cdot \mathbf{n} / \mathbf{k}$. The tensor $\widetilde{D}_{i j}$, given by Eq. (5), is the most general form with respect to the condition of incompressibility of the system under consideration and contains two dimensionless free parameters $\alpha_{1}$ and $\alpha_{2}$. The positiveness of the correlator tensor $D_{i j}$ leads to restrictions on the above parameters, namely, $\alpha_{1}>-1$ and $\alpha_{2}>-1$.

It is mentioned in $[1,3]$ that the system (1) (with zeroth both $\tilde{\mathbf{f}}$ ) is unstable and in QFT formulation the divergence proportional to the UV cutoff $\Lambda$ can appear in the Green function $\left\langle\mathbf{b}^{\prime} \mathbf{b}\right\rangle$. It acquires the form of $\mathbf{b}^{\prime} \operatorname{rot} \mathbf{b} \Lambda$. The $\Lambda$ - UV divergence generates the instability of the theory, that causes an exponential growth in time of the corresponding response function. Therefore, its direct insertion into the action is not allowed and we have to find an effective way to eliminate it. So, one must consider the new vacuum state with a non-vanishing mean value of $\langle\mathbf{b}\rangle \equiv \mathbf{C} \neq 0$. [1]. In QFT the appearance of non-zero vacuum value of field is associated with spontaneous symmetry breaking [34]. The value of spontaneous mean field is determined from re- 
quirement of minimum of potential energy at the tree level. The $\Lambda$-divergence can be eliminated by means of the shift of $\mathbf{b}$ by the value of spontaneous homogeneous magnetic field, namely $\mathbf{b}(x) \rightarrow \mathbf{b}(x)+\mathbf{C}$. This shift in the system (1) with zeroth both $\widetilde{\mathbf{f}}$ produces the new non-vanishing terms

$$
\begin{gathered}
\tilde{\mathbf{f}}^{v}=(\mathbf{C} \nabla) \mathbf{b}+\lambda_{1} \mathbf{C}(\mathbf{n} \nabla)(\mathbf{n b})+\lambda_{2} 2 \mathbf{n}(\mathbf{n} \nabla)(\mathbf{C b})+ \\
+\lambda_{3} \mathbf{n}(\mathbf{C} \nabla)(\mathbf{n b})+\lambda_{4} 2 \mathbf{n}(\mathbf{n C})(\mathbf{n} \nabla)(\mathbf{n b}) \\
\widetilde{\mathbf{f}}^{b}=(\mathbf{C} \nabla) \mathbf{v}
\end{gathered}
$$

with new parameters $\lambda_{i}(i=1, \ldots, 4)$.

\section{THE RENORMALIZATION}

The complete unrenormalized action of the anisotropically forced MHD turbulence $[18,19]$ with the shifted $\mathrm{MF}(\mathbf{b} \rightarrow \mathbf{b}+\mathbf{C})$ [3] can be obtained using $\widetilde{\mathbf{f}}$ and $\widetilde{\mathbf{f}}$ in the stochastic MHD equations (1). Using the standart formalism, the stochastic problem (1) with correlator (4) can be transformed into the field theoretical model of fields $\Phi=\left\{\mathbf{v}, \mathbf{b}, \mathbf{v}^{\prime}, \mathbf{b}^{\prime}\right\}$ where $\mathbf{v}^{\prime}, \mathbf{b}^{\prime}$ are the auxiliary incompressible fields with the action (see $[1,36]$, for details):

$$
S(\Phi)=\frac{1}{2} \mathbf{v}^{\prime} D \mathbf{v}^{\prime}+\mathbf{v}^{\prime} \hat{L}_{v}+\mathbf{b}^{\prime} \hat{L}_{b}
$$

Hereafter in the similar expression the integration over corresponding variables ( $\mathbf{x}, t$ in this case) and the traces over the vector indices are implied. As it is usual in QFT, the action (8) is considered to be unrenormalized with the bare constants marked by the subscript " 0 ". The basic objects of the study are the Green functions of the fields $\Phi$ (i. e., the correlation functions and response functions in the terminology of the original problem (1)). They can be determined as functional derivatives with respect to external sources $A=\left\{A^{v}, A^{b}, A^{v^{\prime}}, A^{b^{\prime}}\right\}$ of the generating functional $G(A)=$ $\left.\int D \Phi \exp [S(\Phi)+]+A \Phi\right]$, i. e., they are the functional averaged values of the corresponding number of the fields $\Phi$ with a weight $\exp [S(\Phi)]$.

In the Fourie representation one obtains:

$$
\begin{aligned}
& S=\frac{1}{2} v_{i}^{\prime} \widetilde{D}_{i j} v_{j}^{\prime}+v_{i}^{\prime} V_{i j l} v_{j} v_{l}-v_{i}^{\prime} W_{i j l} b_{j} b_{l}-b_{i}^{\prime} U_{i j l} b_{j} v_{l}+ \\
& +\frac{1}{2} v_{i}^{\prime}\left[\left(i \omega-\kappa-\kappa \chi_{1} \xi^{2}\right)+\kappa\left(-\chi_{2} n_{i} n_{j}-\chi_{3} \xi^{2} n_{i n_{j}}\right)\right] v_{j}+ \\
& +\frac{1}{2} v_{i}\left[\left(-\mathrm{i} \omega-\kappa-\kappa \chi_{1} \xi^{2}\right)+\kappa\left(-\chi_{2} n_{i} n_{j}-\chi_{3} \xi^{2} n_{i} n_{j}\right)\right] v_{j}^{\prime}+ \\
& +\frac{1}{2} b_{i}^{\prime}\left[\left(\mathrm{i} \omega-u \kappa-u \kappa \tau_{1} \xi^{2}\right)+u \kappa\left(-\tau_{2} n_{i} n_{j}-\tau_{3} \xi^{2} n_{i} n_{j}\right)\right] b_{j}+
\end{aligned}
$$




$$
\begin{gathered}
+\frac{1}{2} b_{i}\left[\left(-\mathrm{i} \omega-u \kappa-u \kappa \tau_{1} \xi^{2}\right)+u \kappa\left(-\tau_{2} n_{i n_{j}}-\tau_{3} \xi^{2} n_{i n_{j}}\right)\right] b^{\prime}{ }_{j}+ \\
+\frac{1}{2} v^{\prime}{ }_{i}\left[\mathrm{i} \gamma+\mathrm{i}\left(\gamma \lambda_{3}+2 \lambda_{4} \gamma_{n} \xi\right) n_{i} n_{j}+\mathrm{i}\left(\lambda_{1} \xi C_{i} n_{j}+2 \lambda_{2} \xi n_{i} C_{j}\right)\right] b_{j}+ \\
+\frac{1}{2} b_{i}\left[-\mathrm{i} \gamma-\mathrm{i}\left(\gamma \lambda_{3}+2 \lambda_{4} \gamma_{n} \xi\right) n_{i} n_{j}-\mathrm{i}\left(\lambda_{1} \xi n_{i} C_{j}+2 \lambda_{2} \xi C_{i} n_{j}\right)\right] v^{\prime}{ }_{j}+ \\
+\frac{1}{2} b^{\prime}{ }_{i}[\mathrm{i} \gamma] v_{j}+\frac{1}{2} v_{i}[-\mathrm{i} \gamma] b^{\prime}{ }_{j}
\end{gathered}
$$

where $\kappa=v k^{2}, \gamma=(\mathbf{C k}), \gamma_{n}=(\mathbf{C n})$ and the vertexes are:

$$
\begin{gathered}
V_{i j l}=\mathrm{i}\left(k_{j} \delta_{i l}+k_{l} \delta_{i j}\right), \quad U_{i j l}=\mathrm{i}\left(k_{j} \delta_{i l}-k_{l} \delta_{i j}\right), \quad W_{i j l}=V_{i j l}+q, \\
\mathrm{i}\left(\lambda_{1} k \xi\left(n_{j} \delta_{i l}+n_{l} \delta_{i j}\right)+2 \lambda_{2 k} \xi n_{i} \delta_{j l}+\lambda_{3} n_{i}\left(k_{j} n_{l}+n_{j k_{l}}\right)+2 \lambda_{4} k \xi n_{i} n_{j} n_{l}\right) .
\end{gathered}
$$

Inversion of the quadratic part of (9) leads to the Green functions (propagators). In general, the Green functions $\langle\mathbf{v v}\rangle,\langle\mathbf{v b}\rangle,\langle\mathbf{b v}\rangle,\langle\mathbf{b b}\rangle$ are expected to be linear combinations of the projectors $P_{i j}, R_{i j} \equiv P_{i r} n_{r} n_{s} P_{s j}, P_{i r} C_{r} n_{s} P_{s j}$, $P_{i r} n_{r} C_{s} P_{s j}, P_{i r} C_{r} C_{s} P_{s j}, Q_{i j}, Q_{i r} n_{r} n_{s} P_{s j}, Q_{i r} C_{r} n_{s} P_{s j}, Q_{i r} n_{r} C_{s} P_{s j}, Q_{i r} C_{r} C_{s} P_{s j}$, $P_{i r} n_{r_{s}} Q_{s j}, P_{i r} n_{r} C_{s} Q_{s j}, P_{i r} C_{r} n_{s} Q_{s j}$, and $P_{i r} C_{r} C_{s} Q_{s j}$. They all are necessary to closing the group of projectors. Analogically, the Green functions $\left\langle\mathbf{v}^{\prime} \mathbf{v}\right\rangle$, $\left\langle\mathbf{v}^{\prime} \mathbf{b}\right\rangle,\left\langle\mathbf{b}^{\prime} \mathbf{v}\right\rangle,\left\langle\mathbf{b}^{\prime} \mathbf{b}\right\rangle$ are expected to be linear combinations of the first five above projectors. In the case of the weak anisotropy limit one can remain the terms of only the first order with respect to all parameters and one can leave out all the terms containing the parameters $\chi_{3}, \tau_{2}, \tau_{3}$, and all $\lambda_{i}$, because these vanish in the fixed RNG points [2]. As a result, one obtains:

$$
\begin{gathered}
\left\langle v_{i} v_{j}\right\rangle=a_{1} P_{i j}+a_{2} R_{i j}+a_{6} Q_{i j}+a_{7} Q_{i r} n_{r} n_{s} P_{s j}+a_{11} P_{i r} n_{r} n_{s} Q_{s j}, \\
\left\langle b_{i} v_{j}\right\rangle=b_{1} P_{i j}+b_{2} R_{i j}+b_{6} Q_{i j}+b_{7} Q_{i r} n_{r} n_{s} P_{s j}+b_{11} P_{i r} n_{r} n_{s} Q_{s j}, \\
\left\langle v_{i} b_{j}\right\rangle=c_{1} P_{i j}+c_{2} R_{i j}+c_{6} Q_{i j}+c_{7} Q_{i r} n_{r} n_{s} P_{s j}+c_{11} P_{i r} n_{r} n_{s} Q_{s j}, \\
\left\langle b_{i} b_{j}\right\rangle=d_{1} P_{i j}+d_{2} R_{i j}+d_{6} Q_{i j}+d_{7} Q_{i r} n_{r} n_{s} P_{s j}+d_{11} P_{i r} n_{r} n_{s} Q_{s j}, \\
\left\langle v^{\prime}{ }_{i} v_{j}\right\rangle=x_{1} P_{i j}+x_{2} R_{i j}, \\
\left\langle b^{\prime}{ }_{i} v_{j}\right\rangle=y_{1} P_{i j}+y_{2} R_{i j}, \\
\left\langle v^{\prime}{ }_{i} b_{j}\right\rangle=z_{1} P_{i j}+z_{2} R_{i j}, \\
\left\langle b^{\prime}{ }_{i} b_{j}\right\rangle=t_{1} P_{i j}+t_{2} R_{i j} .
\end{gathered}
$$

The coefficients in the set (10) have rather complicated form and they linearly depend on five small parameters $\chi_{1}, \chi_{2}, \tau_{1}, \alpha_{1}, \alpha_{2}$. All above Green functions must be calculated for the gyrotropic MHD renormalization, namely, their divergent parts of possessing poles $\sim \varepsilon^{-1}$ in $\varepsilon=0$. These parts are needed for theory renormalization and they are proportional to the second order in momentum unit. This question is not discussed here. In the dynamo theory the linear part of the Green function $\left\langle b_{i} b_{j}\right\rangle$ plays a crucial role [3]. 


\section{CONDITION FOR THE HOMOGENEOUS MAGNETIC FIELD}

The calculation of the Feynman diagram set $\left\langle b_{i} b_{j}\right\rangle$, beside the second-order part, gives also the linear part (in momentum unit) which corresponds to $\Lambda$ - UV divergent rotor part and it must be vanished. The calculation can be performed only under the condition that the homogeneous magnetic field $\mathbf{C}$ direction is parallel to the anisotropy direction $\mathbf{n}$. It yields a strong requirement for possible $\mathbf{C}$ direction, $\mathbf{C}=\mathbf{n}|C|$. Its absolute value follows from the condition of the rotor part vanishing, namely, in the case of the weak anisotropy limit one obtains:

$$
\begin{array}{r}
\left\langle b^{\prime}{ }_{i} b_{j}\right\rangle \approx \mathrm{i} \varepsilon_{i j l} \frac{k_{l}}{k} \frac{\pi g u \rho}{12(1+u)^{2} \sqrt{u}}\{-10(1+u)(3 \pi|\mathbf{C}|-8 \sqrt{u} \Lambda v)+ \\
\left.+[5 \pi(1+3 u)|\mathbf{C}|-16 u \sqrt{u} \Lambda v] \tau_{1}+[5 \pi(5+3 u)|\mathbf{C}|-16 \sqrt{u}(2+u) \Lambda v] \chi_{1}\right\} .
\end{array}
$$

As a result, the requirement of the vanishing of this term yields the value of spontaneous field

$$
\mathbf{C}=\mathbf{n} \frac{4 \sqrt{u} \Lambda v}{45 \pi(1+u)}\left[30(1+u)+(5+9 u) \tau_{1}+(13+9 u) \chi_{1}\right] .
$$

Note that in isotropic turbulence the presence of arbitrary small gyrotropy $\rho$ in Eq. (5) leads to the mean field $\mathbf{C}$ generation [3], but its direction can not be determined (the direction is spontaneous). In contrary, the presence of a small anisotropy of energy forcing into the helical turbulent system leads to the determined direction of $\mathbf{C}, \mathbf{C} \| \mathbf{n}$. In the limit of zero anisotropy $\left(\tau_{1}=\chi_{1}=0\right.$ ), Eq. (12) holds only as scalar equation and $|\mathbf{C}|$ acquires

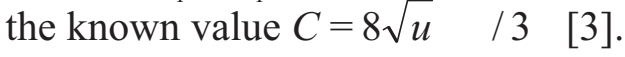

\section{CONCLUSIONS}

In the paper, the phenomenon of the magnetic field generation in astrophysical environment is studied. The statistical dynamo model is constructed for helical weakly anisotropic full developed MHD turbulence in the framework of the quantum field renormalization group approach. It is shown that the renormalization leads to arising of ultra-violet divergence in rotor part of the magnetic field Green function $\left\langle b^{\prime}{ }_{i} b_{j}\right\rangle$ which must be eliminated from the theory. The weak helical anisotropic turbulence is then stabilized by the self-generated magnetic field $\mathbf{C}$ whose value is calculated. The direction of the magnetic field is connected with unique existing vector $\mathbf{n}$ describing the anisotropic turbulence forcing, $\mathbf{C} \| \mathbf{n}$.

Note that the renormalized Green function is obtained which is finite as $\Lambda \rightarrow \infty$ formally, as it is usual in the field theory. But in real problems a natural cutoff really exists. In the developed turbulence the Kolmogorov dissipative length $l_{D}=\Lambda^{-1}$ plays the role of a minimal scale. This length can 
be expressed in terms of basic phenomenological parameters - viscosity $v$ and energy dissipation rate $\varepsilon$. Then from (13) and simple dimensional considerations one obtains $|\mathbf{C}| \propto(v \varepsilon)^{1 / 4}$ — order of magnitude of the spontaneous field [36].

Acknowledgements. M. Stehlik gratefully acknowledges the hospitality of the staff of the Main Astronomical Observatory of NAS of Ukraine (Kyiv). The paper was prepared in the course of the realization of the project ITMS No. 26220120029 based on the supporting operational Research and development program financed from the European Regional Development Fund and also supported by SAS, VEGA project No. 2/0081/10 and 2/0173/09.

1. Adzhemyan L. Ts., Antonov N. V., Vasiliev A. N. The field theoretic renormalization group in fully developed turbulence. - London: Gordon and Breach Sci. Publ., 1999. - 202 p.

2. Adzhemyan L. Ts., Hnatich M., Horvath D., Stehlik M. Infrared properties of an anisotropically driven MHD turbulence // Int. J. Mod. Phys. B.-1995.-9.- N 26.P. 3401-3419.

3. Adzhemyan L. Ts., Vasiliev A. N., Gnatich M. Turbulent dynamo as spontaneous symmetry breaking // Theor. Mat. Phys._-1987._72, N 3.-P. 940-950.

4. Blackman E., Field G. Constraints on the magnitude of alpha in dynamo theory // Astrophys. J.-2000.-534, N 2.-P. 984-988.

5. Blackman E., Field $G$. New dynamical mean-field dynamo theory and closure approach // Phys. Rev. Lett.-2002.-89.-P. 265007.

6. Blackman E., Ji H. Laboratory plasma dynamos, astrophysical dynamos, and magnetic helicity evolution // Mon. Notic. Roy. Astron. Soc._2006._369, N 4.-P. 1837 1848 .

7. Brandenburg $A$. The inverse cascade and nonlinear alpha-effect in simulations of isotropic helical hydromagnetic turbulence // Astrophys. J.—2001._550, N 2.-P. 824840.

8. Brandenburg A., Donner K. J. The dependence of the dynamo alpha on vorticity // Mon. Notic. Roy. Astron. Soc.—1997.-288, N 2.-P. L29-L33.

9. Brandenburg A., Sokoloff D. Local and nonlocal magnetic diffusion and alpha-effect tensors in shear flow turbulence // Geophys. Astrophys. Fluid Dyn._2002.-96.P. 319-344.

10. Chou H. The dependence of dynamo alpha-effect on Reynolds numbers, magnetic Prandtl number, and the statistics of magnetohydrodynamic turbulence // Astrophys. J. -2001.-552, N 2.-P. 803-820.

11. Davidson P. A. Turbulence. — Oxford: University Press.—-2004._657 p.

12. Donner R., Seehafer N., Sanjuan M. A. F., Feudel F. Low-dimensional dynamo modelling and symmetry-breaking bifurcations // Phys. D._2006.-223.-P. 151-162.

13. Field G., Blackman E., Chou H. Nonlinear alpha-effect in dynamo theory // Astrophys. J.-1999.-513, N 2.-P. 638-651.

14. Field G., Rogers R. D. Radiation from magnetized accretion disks in active galactic nuclei // Astrophys. J.-1993._403, N 1.-P. 94-109.

15. Galeev A. A., Rosner R., Vaiana G. S. Structured coronae of accretion disks // Astrophys. J.-1979.-229, N 1.-P. 318-326.

16. Haugen N. E. L., Brandenburg A., Dobler W. Is nonhelical hydromagnetic turbulence peaked at small scales? // Astrophys. Lett._2003._597._P. L141—L144.

17. Hnatich M., Horvath D., Stehlik M. Koncepciya krupno- i melkomasshtabnykh stokhasticheskikh silovykh polei v MGD turbulentnosti // Magnitnaya Gidrodinami- 
B. A. SHAKHOV et al.

ka.-1997.-33.-P. 266-274. (In Russian)

18. Hnatich M., Jonyova E., Jurcisin M., Stehlik M. Stability of scaling regimes in $d \geq 2$ developed turbulence with weak anisotropy // Phys. Rev. E._2001._64._-P. 016312$1-10$.

19. Jurcisin M., Stehlik M. D-dimensional developed MHD turbulence: double expansion model // J. Phys. A: Math. Gen._2006._39.-P. 8035-8050.

20. Kazancev A. P. Usilenie magnitnogo polya v provodyashchei srede // Zh. Eksper. Theor. Fiz.—1968.-26.-P. 457-460. (In Russian)

21. Kida S., Yanase S., Mizushima J. Statistical properties of MHD turbulence and turbulent dynamo // Phys. Fluids A.-1991.-3.-P. 457-465.

22. Krause F., Rädler $K$.-H. Mean-field magnetohydrodynamics and dynamo theory. New York: Pergamon Press, 1980.-320 p.

23. Lithwick Y., Goldreich P. Compressible magnetohydrodynamic turbulence in interstellar plasmas // Astrophys. J._2001._562, N 1._P. 279-296.

24. Maron J., Goldreich P. Simulations of incompressible magnetohydrodynamic turbulence // Astrophys. J.—2001._554, N 2._P. 1175-1196.

25. Martin P. C., Siggia E. D., Rose H. A. Statistical dynamics of classical systems // Phys. Rev. A.-1973.-8, N 1.—P. 423-437.

26. Moffatt H. K. Magnetic field generation in electrically conducting fluids. - Cambridge: Univ. Press, 1978.-339 p.

27. Montgomery D. C., Matthaeus W. H., Milano L. J., Dmitruk P. Apparent suppression of turbulent magnetic dynamo action by a dc magnetic field // http://lanl.arxiv.org/abs/ physics/0202027.-2002.

28. Parker E. N. Cosmical Magnetic Fields.-Oxford: Clarendon Press, 1979.—608 p.

29. Pouquet A., Frish U., Leorat J. Strong MHD helical turbulence and the nonlinear dynamo effect // J. Fluid Mech._1976._77, N 2.—P. 321-354.

30. Rädler K.-H., Brandenburg A. Alpha-effect dynamos with zero kinetic helicity // Phys. Rev. E.-2008.-77, N 2.-P. 026405.

31. Rädler K.-H., Kleeorin N., Rogachevskii I. The mean electromotive force for MHD turbulence: The case of a weak mean magnetic field and slow rotation // Geophys. Astrophys. Fluid Dyn.-2003._-97, N 3.-P. 249-274.

32. Rädler K.-H., Rheinhardt M. Mean-field electrodynamics: Critical analysis of various analytical approaches to the mean electromotive force // Geophys. Astrophys. Fluid Dyn.-2007.— (http://xxx.lanl.gov/abs/astro-ph/0606267)

33. Schekochihin A. A., Cowley S. C. Turbulence and magnetic fields in astrophysical plasmas // http://xxx.lanl.gov/abs/astro-ph/0507686.-2005.

34. Shakhov B., Stehlik M. The $\alpha$-effect and proton acceleration in the solar wind // Kinematics Phys. Celestial Bodies.-2008.—24, N 1.-P. 28-34.

35. Sur S., Subramanian K., Brandenburg A. Kinetic and magnetic alpha effects in nonlinear dynamo theory // Mon. Notic. Roy. Astron. Soc._2007._-376, N 3._- P. 1238 1250.

36. Vasiliev A. N. The field theoretic renormalization group in critical behavior theory and stochastic dynamics. — Boca Roaton: Chapman and Hall/CRC, 2004.—774 p.

37. Verma M. K., Lessinnes T., Carati D., et al. Dynamo transition in low-dimensional models // Phys. Rev. E._2008.-76.-P. 036409.

Received 27.10.12 